\title{
Analysing group interaction in focus group research: Impact on content and the role of the moderator
}

\author{
Mette Gronkjir, Tine Curtis, Charlotte de Crespigny \& Charlotte Delmar
}

\begin{abstract}
Interaction between group participants is considered the distinct advantage and hallmark of focus group research. It is therefore necessary to include the social interaction dynamics in analysing focus group data. Little information is however available on analysis of the social interaction in the group and the analytical outcome for the content of the data. This paper contributes to the discussion of the value of participant interaction in focus group research by analysing sequences of interaction collected recently during a research project. This project utilized focus groups to investigate the perceptions and meanings of alcohol use in Denmark. As a frame for analysing group interaction, elements of conversation analysis were used. The aim of this paper is to illustrate group interaction and its impact on the content of focus group data, and highlight the role and some of the challenges posed by group interaction for moderating the focus group discussion. The interaction analyses led to the construction of four interactional events: Negotiating and constructing normality in interaction, disagreement and/or consensus, homogeneity and the impact on interaction and content, and coming to and making sense of a dead-end (including the risk of hierarchical issues). The interactional events are followed by considerations on the impact they may have on the role of the moderator.
\end{abstract}

Keywords: focus group; group interaction; moderator role; conversation analysis

Please cite this article as:

Grønkjær, M., Curtis, T., de Crespigny, C. \& Delmar, C. (2011). Analysing group interaction in focus group research: Impact on content and the role of the moderator. Qualitative Studies, 2(1): 16-30.

\section{Introduction}

Focus groups are well established as a legitimate data collection method within the qualitative research tradition. The method was originally developed in the field of market research, but has become frequently used in social science, health and related disciplines. The rationale behind the use of focus groups is that knowledge is created through the diverse experiences and forms of knowledge of, and interaction between, participants. Focus group participants provide an audience for each other, which encourages a greater variety of communication, and therefore different contents, than other qualitative methods of data collection (Kitzinger, 1995).

The main advantage of focus groups involves how group interactions can reveal and highlight the participants' perceptions, attitudes, thinking, and framework of understanding, as well as identifying group norms, sub-cultural and cultural values (Kitzinger, 1995; Kitzinger, 1994; Krueger \& Casey, 2000). Findings of focus group research, however, often report results using quotations from one individual at a time. This may give the impression that individual viewpoints can be isolated from the interaction between the group participants (Duggleby, 2005; Wibeck, Dahlgren, \& Oberg, 2007). Halkier (2010) stresses the necessity of including the social interaction dynamics in analysing focus group data. Although the literature deals with focus group interaction as part of moderator questions, group composition and introduction to focus groups (Rabiee, 2004; Plummer-D'Amato, 2008; Bloor, Frankland, Thomas, \& Robson, 2001), less is available on the analysis of the social interaction in the group and the analytical 
outcome for the contents of the data (Halkier, 2010). This view is supported by other authors who claim that even though interaction is the hallmark of focus group research, the interaction has seldom been analysed or discussed in research based on empirical material collected from focus groups (Hydén \& Bülow, 2003). Wibeck et al. (2007) further claim that researchers may capture and take advantage of the interaction itself when analysing the data, rather that treating interaction as merely a tool for efficient data collection. Successful group interactions between focus group members rely to a large extent on the moderator (McLafferty, 2004; Morgan, 1997; Krueger et al., 2000; Curtis \& Redmond, 2007; Redmond \& Curtis, 2009; Plummer-D'Amato, 2008; Wibeck et al., 2007; Bloor et al., 2001; Barbour, 2007). Most authors identify group interaction as the distinct advantage of focus groups, but it is also one area that presents a number of challenges to the researcher (Clark, Maben, \& Jones, 1996). Thus, in developing focus group methodology on group interactions, the role and challenges of the moderator on group interaction should be considered and discussed. Moderator challenges on group interaction need to be recognized, addressed and carefully dealt with (Warr, 2005), as there is a risk of collecting data of poor quality, and consequently less trustworthy results.

This paper provides a contribution to the discussion on interaction in focus group research by analysing sequences of group interaction that were recently collected during a research project where focus groups were used to investigate the perceptions and meanings of alcohol use in Denmark. As a frame for analysing group interaction, elements of conversation analysis are used (Silverman, 2006; Potter, 1996; Puchta \& Potter, 2004). The paper aims to illustrate group interaction and its impact on the content of focus group data, and highlight the role and some of the challenges group interaction pose on moderating the focus group discussion.

The next section provides an overview of the particular study, including background and method. This is succeeded by a brief description of conversation analysis followed by analyses of group interaction sequences (interactional events) from this particular study. Each interactional event is succeeded by considerations on the role of the moderator in such events.

\section{A research project on alcohol use as example}

This paper draws upon empirical examples from the research project 'Alcohol use in Denmark: drinking contexts and the meanings and perceptions of alcohol use from a public health perspective'. The background for this research is that alcohol consumption levels in Denmark are high. The prevalence of people who drink alcohol above the recommended levels of low risk to health is increasing, which is reflected in alcohol related causes of premature mortality, morbidity and lowered life expectancy (WHO, 2004). Although a significant amount of literature exists on the amount and frequency of alcohol consumption in Denmark, there is far less concern with the cultural and contextual influences on alcohol use. For effective public health and health promoting initiatives, normality perspectives and cultural factors are important in understanding not just individuals' behaviour such as risky drinking, but also the external influences on, and conditions in which, this occurs (Rose, 1992). Therefore this study sought to reveal and describe the cultural and contextual influences on Danes' alcohol use from a public health perspective. The overall study used a sequential mixed methods approach (Creswell, 2003), and consisted of an initial quantitative study using national survey data, followed by a qualitative study using focus group discussions. The aim of the overall study was to create new knowledge of alcohol use in Denmark that can be applied in the development of future public health policies and strategies, including health promotion, within the alcohol area. 


\section{Participants}

The study consisted of five focus group interviews between Danish participants. The focus groups were each constructed based on the following age groups: $16-20 ; 21-34 ; 35-44 ; 45-64$; and 65-82. The groups consisted of both men and women. There were five to six participants in each group (a total of 27). All names reported below are pseudonyms.

\section{Sampling and recruitment}

Participants were purposely sampled through the use of selected gate keepers who were able to recruit participants with diverse characteristics to ensure variation in gender, social and professional backgrounds. This approach was aimed at ensuring both homogeneity and heterogeneity in the group construction (Morgan, 1997). Thus, the focus groups were characterized by age as the factor of homogeneity to ensure some degree of commonality among participants, but with sufficient variation to allow for contrasting opinions (Krueger et al., 2000).

\section{Focus groups}

A theme guide was developed and used to guide the discussion. The opening question in each of the five focus groups was: "what is the first you come to think of when I say alcohol and alcohol use in Denmark"? This was succeeded by a discussion of the following themes: drinking contexts; the "normal" use of alcohol; the meaning of alcohol use and public health perspectives in relation to alcohol use. The group discussions were audio-recorded onto a mini-disc and transcribed ad verbatim for analysis. Data analysis was performed using NVivo software and two major themes emerged: Acceptance and Expectance and Drinking Contexts and the Legitimacy of Alcohol Use. The results are under preparation for publication.

\section{Conversation analysis}

The analysis in this paper is inspired by, and uses elements of, conversation analysis (Silverman, 2006; Potter, 1996; Puchta et al., 2004) to analyse focus group interaction from the abovementioned research project. As such, the paper is not interested in making in-depth descriptions of conversation analysis, but rather to contribute to the discussion of participant interaction in focus group research using elements of conversation analysis.

Conversation analysis is concerned with actions and interactions as social phenomena. It studies talk in interaction and considers conversation as action taking place between actors (Potter, 1996; Puchta et al., 2004). Conversation analysts claim that conversation is far from disorderly; in fact it is incredibly orderly. The principal ambition of conversation analysis is to reveal and account for this orderliness (Potter, 1996). Thus, conversation analysis is based on the attempt to describe people's methods for producing orderly social interaction (Silverman 2006; Potter 1996). As with other methodological tools, e.g. discursive psychology, conversation analysis can be employed to systematically analyse focus group interaction data (Puchta et al., 2004). While discursive psychology goes beyond the conversational situation itself and attempts to analyse how situational interaction draws upon, negotiates, moderates and changes the larger discursive repertoires, conversation analysis is concerned with the orderliness, structure and sequential patterns of interaction (Silverman 2006; Potter 1996). By viewing talk as a form of social action and paying attention to the sequence and structure of talk, conversation analysis can assist the researcher in getting access to the construction of meaning and social action being performed by the group participants (Barbour 2007; pg. 37). Conversation analysis can also assist in analysing how the contents of norms are negotiated within the focus groups (Halkier, 
2010). Thus, this methodological approach was found relevant for analysing group interaction in the abovementioned project.

Conversation analysis offers a range of tools for analysing interaction between participants in focus groups. In this analysis, we have used one of the simplest, but most fundamental analytical tools from conversation analysis; adjacency pairs (Potter, 1996). Adjacency pairs are fundamental to interaction and can be thought of as the basic glue of conversation. The rationale is that many actions in conversation are linked together; when one specific action is undertaken it is likely to lead to a second specific action (Potter, 1996). Adjacency pairs bind a conversation together and are thus considered normative relations; for example between questions and answers (Silverman, 2006; Puchta et al., 2004). Given this normative relation, adjacency pairs involve preference organisation (Potter 1996). This means that to a particular utterance, there is a preferred response (i.e. acceptance or agreement) or a dispreferred response (i.e. rejection or disagreement). A particular feature of adjacency pairs is the account. An account concerns the justifications behind the decisions, opinions and actions that participants express in the focus groups; most often characterised by descriptions. In some cases, the process of building accounts and descriptions involve repair. Repair is often used in focus group interaction and is concerned with how participants repair their own and, more delicately, others' talk (Puchta et al., 2004).

Our analyses resulted in a series of events that occurred in the focus group interactions. The events are as follows: Negotiating and constructing normality in interaction, disagreement and/or consensus, homogeneity and the impact on interaction and content, and coming to and making sense of a dead-end (including the risk of hierarchical issues). In the following, the events are illustrated with empirical examples. Each sequence is followed by considerations of the role of the moderator with regard to the specific events.

\section{Negotiating and constructing normality in interaction}

In the study of alcohol use in Denmark, the idea was to investigate the cultural perceptions and meanings of alcohol use, including normality perspectives. In the following extract, the focus group participants aged 65 to 82 are discussing what constitutes normal use of alcohol:

Per: Well, we have already discussed this thing about sharing a bottle of wine for dinner.

Connie: $\quad Y e s$, we have. Because we are sharing a bottle when two people are gathered. But not as much in our home anymore because I have become more moderate .... [Connie mentioned previously that she is on a diet]. So there is always a little drop left in the bottle for the next day, but I only drink one glass.

Per: $\quad$ Not in our house. I have to admit that. We drink a bottle of wine every evening.

Connie: $\quad$ And we have always done that. We have.

Ellen: $\quad$ I too think that a bottle is suitable for two.

Connie: $\quad$ I think so too.

Knud: Well, not every day?

Connie: $\quad$ Yes, every day.

Bodil: $\quad$ No, Friday and Saturday.

Knud: I find it very un-normal if you drink so much every day. I think you have that reminder about the 7 and the 14 [refers to standard drinks/maximum recommended level per week]....

Per: $\quad$ No, well that's not enough. It is 14 and 21. 
Jens: $\quad 14$ and 21

Knud: $\quad$ Oh yes, 14 and 21, yes. Yes, but I think you... And I know that if you say that you are just going to have a glass for dinner every evening. Well, there is not much left in a bottle when you are finished anyway. I know that [laughing], but I still think that you think a little about it if you drink every day. That you want to stay a little under what they recommend.

Moderator: So you are conscious about the recommendations?

Ellen: $\quad$ Yes, I am.

Per: $\quad$ I am not.

Ellen: $\quad$ I take them into consideration.

Per: $\quad$ I have a feeling about it, but I never think about it. As I told.... my wife has an old friend and they were discussing this thing about the 21 drinks a week and he said: Oh god, I am already there by Monday.

[Everyone laughs]

Connie: $\quad$ Yes, there are some who drink more than others.

Per: $\quad$ But conscious about it... I don't think you think about it. I don't think about it. But, you have a feeling of ... where... what ....there are three.... There are three standard drinks in a bottle of wine [sharing a bottle with his wife each night], and times 7, then you are at 21. And then the rest during the week, right. So then you are up to about 26, 28 per week. I don't think it is harmful when it is wine. I think it is worse when it is strong spirits.

Connie: $\quad$ Yes, I think so too. Because I think it is rare that we drink strong spirits.

In this extract, adjacency pairs are exchanged when participants share their experiences and discuss their perceptions of the normal use of alcohol. Initially, Per and Connie express agreement when they refer to their previous discussions on what they find normal (sharing a bottle of wine every evening). Ellen contributes with a preferred reaction in that she agrees that a bottle is suitable for two. In response, Knud enters the discussion by questioning the others' opinions and by that he destroys the perceived consensus. This dispreferred response challenges the perceived agreement between Per, Connie and Ellen and initiates a negotiation about normal use of alcohol. The sequence is then characterised by repair when Per corrects Knud on the recommended levels of alcohol (maximum 14 standard drinks per week for women; 21 for men). Although not agreeing to the other group members' view on normal use of alcohol, Knud negotiates his opinion by indicating his understanding ('I know that'). This response may be an attempt to soften the dispreferred reaction. He also laughs when he provides his point about considering the maximum recommended levels, which may make the disagreement less uncomfortable. Moreover, the fact that Knud uses you-form instead of I-form may even indicate the normative character of the interaction; this change of forms is a way of making an account that supports his own view of considering the recommended levels (as you normatively should, according to the health authorities; a preferred response).

In the latter part of the sequence, the group discusses their consciousness about the maximum recommended levels for alcohol use. Per is not conscious about them and accounts for his behaviour by providing an anecdote from his wife's friend that makes the group laugh. The introduction of joking can be seen as one way of making an account or diffusing an uncomfortable situation; but it also functions as an invitation for the other participants to join in. Connie accepts this invitation by giving a comment on what she views as generally understood about there always being some people who drink more than others, and Per returns to the initial question by using descriptions to account for his opinion. In this case, Per justifies 
his own alcohol use by listing the amount of alcohol he would normally drink on a weekly average. Despite revealing that he drinks alcohol above the recommended levels, he justifies his drinking by stating that as long as it is wine, and not strong spirits, it is not harmful. He receives a preferred response from Connie who argues her case by saying that we (she?) rarely drink spirits (as opposed to wine). Thus, they use their own alcohol behaviour to construct the idea that wine is not as harmful as spirits.

The interaction above illustrates that there is diversity in the group with regard to the perceived normality of use and conforming to the recommended levels. As expected, there are many and varied patterns of drinking and thus a variety of perceptions of what constitute normal drinking behaviour. The exchanges of adjacency pairs; the agreements and disagreements, the accounts and the way they are built provide rich data on the subject. What is interesting about this sequence, and quite relevant for public health purposes, is the way the participants account for their use of alcohol and negotiate their opinions. Knud has to account for his opinion by laughing and expressing that he understands in order to prove to the other group members that it is okay to be conscious about the recommended levels. This example of interaction is quite indicative of one of the major public health challenges to alcohol; that considering and/or living by the national recommended levels is not the norm. And if you do, you have to explain why. The use of laughing during the negotiations may also indicate a non-serious understanding of alcohol use.

Besides highlighting the importance of paying attention to the accounts and negotiations between participants for analytical purposes, the above extract also accentuates the role of the moderator. The moderator role in this case involved thoroughly considering their own responses to the participants' interactions and responses in order to achieve the rich data set: i.e. bracketing public health knowledge on alcohol and remaining in the researcher role, instead of, for example, moving into the role of a health professional and interrupting the interaction by informing participants of the recommended low risk drinking levels. It was crucial for the moderator to signal approval and remain open e.g. by keeping eye contact and nodding; listening and not commenting on the details of the discussion, and, if necessary, elaborating on aspects relevant to the study: for example by asking participants to expand on their views rather than questioning them. In this case, asking participants to consider their consciousness about the recommended levels. It was also necessary for the moderator not to seek consensus as participants have divergent views which require analysis and reporting in their own right.

\section{Consensus and/or disagreement}

In this study on alcohol use, participants displayed diverse perceptions about alcohol use being healthy or not. Here follows an extract from the group aged 45 to 64 :

Michael: $\quad$ I've never thought that it was healthy to smoke ... you have also said a few times that a glass of red wine is healthy...

Tove: $\quad$... well yes, we are told...

Michael: $\quad$... cholesterol decreasing and so forth and I guess that ...

Hanne: $\quad$ Yes, but we don't stick with that. None of us do.

Michael: $\quad$ But we . . because I guess it [alcohol] is not that healthy. It may be in relation to cholesterol, but in relation to accidents, you can't continue to claim that ...

Lene: $\quad$... that it is healthy.

Michael: $\quad$ Yes, that it is healthy.

Hanne: $\quad$ I think you could eat a lot of different things that are just as healthy as red wine. 
Well I believe it is healthy if you drink a glass or two a day. Just like the TVdoctor says. Why wouldn't you believe that?

Erik: $\quad$ I CERTAINLY don't think so. Not every day. I don't think so

Hanne: No, alcohol is metabolized in the liver. It is not good for the liver to work overtime either.

Tove: Well is that overtime, one or two glasses? One or two standard drinks?

This extract illustrates an example of disagreements between participants and the impact the interaction has on the content of the discussion. Michael opens the sequence by questioning the health benefits of alcohol by comparing it to smoking and directs his reaction to Tove who previously said that a glass of red wine is healthy. In this adjacency pair, Tove overlaps Michael's utterance and elaborates on her previous statement by creating an account (well yes, we are told). Michael continues his initial utterance using knowledge on alcohol's effect on cholesterol levels to create an account. Hanne changes the direction of the conversation; leading Michael to negotiate the idea that alcohol might not be as healthy after all. This is followed by Tove who obviously disagrees with their opinions and uses the TV-doctor, a health authority from national television, as assistance in making her account. Erik provides a dispreferred response; indicating that he strongly ('CERTAINLY') disagrees. Hanne reacts with a preferred reaction in that she agrees and provides a physiological explanation as an account. Despite Hanne's account, in this case scientific knowledge about alcohol's effect on the liver, Tove continues to question the argument; a dispreferred response to Hanne's claim. Thus, the participants' diverse understandings of alcohol and health functions as a catalyst, not only in keeping the discussion going, but also in moving their perceptions from uncertainty to certainty about the health benefits of alcohol.

The sequence above is characterised by active interaction; the participants obviously disagree and they question one another. The discussion concerned the so-called alcohol preventive paradox indicating that there are both beneficial and harmful effects of alcohol on health (Gronbaek, 2001). Being characterised through lack of consensus, this interaction sequence is important for several reasons. The accounts used in the exchange of adjacency pairs reflect some of the public's understandings of alcohol and health/ill-health. It illustrates an uncertainty ('I guess it is not that healthy') that during the discussion becomes negotiated to certainly not being healthy. The conflicting opinions reveal that alcohol's preventive paradox is vital in people's perceptions. The use of the TV-doctor and continuous questions to the group members' accounts ('well is that overtime...?'), manifest a confidence in the beneficial effect of alcohol on health. The disagreement in the group, and the accounts that participants used to argue their case, illustrated how group interaction closely relates to the contents of the data. Moreover, it reveals diverse and important understandings of alcohol and health that need to be incorporated in the public health discussions and initiatives on alcohol use.

In this case of obvious disagreement, the role of the moderator involves paying close attention to the interactions: Firstly in being able to understand the meanings of the disagreements and secondly, to promote the group dynamics and a friendly atmosphere despite the disagreements. This can be carried out by acknowledging participants' diverse views in the case of disagreements and emphasising to participants that the study is interested in different views on the research topic. In this case, it was important to convey to Tove that by disagreeing with the rest of the group, her views were just as important as theirs. 


\section{Homogeneity and its impact on interaction and content}

Homogeneity in focus group construction is considered essential for group interaction and dynamics, and thus important for the moderator to consider. In this study, age was used to ensure some degree of homogeneity. The following extract illustrates the role homogeneity can play (in this case age homogeneity) for group interactions and thus for the contents of the discussion. This discussion took place among those aged 65 to 82 years:

Moderator: $\quad$...you mentioned the price of alcohol, and that it is less expensive [today] to buy a case of beer? Can you...

Knud: $\quad$ Yes, not in relation to then ... [earlier days]

Per: $\quad$ And if there is not enough wine on the table, we'll just open another one

Connie: $\quad$ Yes, we'll just get some more...

Per: $\quad$... we'll just do that...

Connie: $\quad$... because we all have it. You have wine cellars, right.

All: $\quad$ Yes, yes.

Connie: It is in store.

Per: $\quad$ It must not affect the cosiness.... [to not have wine]

Knud: $\quad$ No, there has to be something.

Ellen: $\quad$ I guess it [wine] is a hobby for a lot of people.

Connie: $\quad$ Yes, to invest in the right wines.

Ellen: $\quad$... yes, and read about it and things like that. People didn't use to do that.

Connie: $\quad$ Yes, wine clubs and so forth, right.

Ellen: $\quad$ Uh the synthetic wine back then. Do you remember? What was is called?

Connie: Ahh, yes Palanca from Brugsen [food store] and then there was Pierre Medard and ...

Jens: $\quad$ The one with the bull ...

Connie: $\quad$ That was awful wine. Now I understand why I didn't like wine then.

Per: $\quad$ The kids were complaining because they couldn't have a bath because there were always three balloons in the bathtub [referring to homemade wine] [Everyone laughs]

Ellen: $\quad$ YES, it smelled like ... it was fermenting.

Connie: $\quad$ Uh yeah, that was awful.

Moderator: $\quad$ Okay, well... let's move on to ...

In contrast to the previous examples of negotiations and lack of consensus, this sequence illustrates interaction characterised by consensus and its impact on the content. In this extract, the group is initially discussing the price of alcohol by comparing it with earlier days and now. The exchanges of adjacency pairs illustrate agreement between participants when they talk about wine; for example when Ellen talks about synthetic wine and Connie accepts the invitation by agreeing and elaborating with descriptions about the wine they drank when they were younger. They end up sharing their experiences from when they either drank synthetic wine or made the wine themselves.

Because they belonged to the same age group, participants were able to recall the price of alcohol years ago and share anecdotes from back then. This sequence of interaction is characterised by an easy flow between group members. The exchanges of adjacency pairs display acceptance of the participants' utterances. This is illustrated by the many instances where participants take over and/or complete each others' sentences; they are eager to share their points and understand each others' utterances. Thus, it is easy for them to complete what 
the other is about to say. The extract also contains very few descriptions that account for diverse opinions on the topic of discussion, which possibly indicated a general agreement between the participants. This indicates that people's perceptions of alcohol are to some extent associated with age and reflects how age as a factor of homogeneity may influence the participants' group interaction and content.

In the above case, where the interaction was characterised by agreement and easy flow, the role of the moderator is, again, to pay close attention to the interaction. It may also be necessary to challenge the participants' accounts to add new dimensions to the discussion or to interrupt discussions in order to increase discussion or change the focus. In such cases it is important to consider the fact that participants have consented to their participation and are using their valuable time in making a study possible. As such, some participants may feel uncomfortable or offended when asked to change direction or let others continue talking. This highlights the challenge and delicate balance between considering both the respect for the individual group members, the need to elicit diverse views and the aim of the research. This balance impacts on group interaction, and so it is important for the moderator to have the skills and ability to sense the situation. They need to "lean back" as appropriate and let the interaction flow with the aim of obtaining essential data, and when appropriate, end or change the direction of discussion.

\section{Coming to and making sense of a dead-end}

The next extract illustrates how the turn-taking in the interaction between participants can come to a dead-end, and the resultant challenge in making sense of it. It also illustrates the importance of the moderator to be able to reflect and make some sense of the participants' responses during the group discussion. The following extract is from the group aged 35 to 44 :

Moderator: Other focus group members have mentioned that it is not okay to say no to a drink...

Palle: But I actually think that this [tendency] starts in the younger years. It is a bit like a challenge: don't you dare to drink? Maybe you have adapted this from then. That it is latent in you: ahhh, come on [have a drink]

Ulla: $\quad$ No damn it, I don't dare that. The first time I was pissed [drunk] I thought I was going to die because I threw up and it was blood. Then I was told afterwards that it was a red soda and I haven't drunk it [red soda] since.

Pause

Moderator: Alright, we have talked about the social aspect of alcohol, about cosiness and so forth. Now I would like to talk about....

In this extract, the moderator's opening question is aimed at helping the group consider preliminary results from other focus group discussions and therefore elaborate on preliminary findings. Palle provides an account by suggesting that other people's expectations that you should drink may come from challenges they experienced in their younger years. Ulla's response is a description of her experiences from the first time she was drunk. This leads to a pause followed by a change of direction from the moderator. This extract is interesting because it seems that Ulla does not follow the discussion path if she felt it was okay to say no to a drink or not. Instead she (explicitly) responded to Palle's account ('don't you dare to drink?'). Ulla's response may be an indication that daring to drink is an important topic for her to discuss or convey; or it may be that she misunderstood the question. It may also be a way for Ulla to express or exemplify her view on this discussion on saying no to a drink. Instead of elaborating on Ulla's response, the moderator closes the discussion by summing up and changing the focus. 
This could be perceived as a dispreferred response in that Ulla is "cut-off" and does not receive a response to her utterance. In fact, not replying to her utterance and changing the topic may be quite a violation of the normative relationship between adjacency pairs. In this case, coming to a dead-end may very well be due to the moderator's ability to pursue the meaning of an implicit response. In hindsight, it would have been relevant to either offer repair by delicately suggesting Ulla may have misunderstood - or at least offering an invitation to elaborate on her statement instead of closing it down.

The extract above can also illustrate the risk of hierarchical issues or power relations due to the asymmetric relation between moderator and participants. As such, Ulla may become intimidated by the lack of response to her statement by the moderator. The risk of hierarchical issues can also be exemplified by the various words and phrases used by participants. For example; Ulla's response above illustrates a descriptive sentence construction whereby she uses everyday language and swearing. In comparison, some other participants were very eloquent; they expressed analytical statements and critically reflected on others' utterances in their groups. Some also knew a lot about the topic under investigation and were at times theoretical in their expressions. They provided information on the latest statistics and theorised about various ideas and perceptions revealed in their group. These participants" "expert knowledge" may have overly influenced the power relations within the group, resulting in other participants feeling intimidated and deciding to withdraw from the discussion.

The examples above illustrate how the moderator role needs to involve a continuous assessment of the interactions between various participants, how their choice of words and phrases might be affecting other members' participation in the group, and what impact homogeneity or heterogeneity might have on the group interaction. This illustrates the need for the moderator to ensure that all focus group participants are able to be heard, including those who may not be as eloquent or comfortable, yet equally important in conveying their messages. Krueger \& Casey (2000) have suggested a moderator conducts a pre-session of small conversation so as to identify the more dominant speakers, and identify participants' body language during this time. The moderator may shift their own non-verbal attention by for example removing eye contact from the dominant talker to the rest of the group and saying: "Thank you for your comment. How do you [the others] feel about this?" Or elaborating on an utterance that potentially possesses implicit or multiple meanings; as for example Ulla's response above.

\section{Discussion and conclusion}

The influence of the social interaction between members of focus groups is often used to argue for the use of focus groups for data collection in particular qualitative research projects. The social interaction has, however, often been neglected in analysing and reporting focus group data; including its impact on eliciting the contents of such data. The literature has emphasized the uniqueness of participants' interaction in focus group research (Duggleby, 2005; Wibeck et al., 2007; Halkier, 2010) and this paper contributes to the discussion by exemplifying interaction data sequences from recent a research project on alcohol use in Denmark.

Using elements of conversation analysis, the authors have analysed a series of interactional events that took place during data collection in five focus groups made up of different age groups: Negotiating and constructing normality in interaction, disagreement and/or consensus, homogeneity and the impact of interaction and coming to and making sense of a dead-end 
(including the risk of hierarchical issues). Each event has been presented followed by considerations regarding the role and responsibility of the moderator in this context.

The analysis of adjacency pairs, including preference organisation, identified how perceived normality regarding drinking alcohol can be negotiated and constructed, i.e., the focus group participants' perceptions of what is normal alcohol use. The analysis revealed deficits in understanding risky and low risk alcohol consumption concerning health, and that considering and/or living by the national recommended levels for low risk alcohol use is not the norm. This supports and elaborates on existing alcohol research (Mandag Morgen \& TrygFonden, 2008; Grønkjær, Vinter-Larsen, Curtis, Grønbæk, \& Nørgaard, 2010) suggesting the importance of public health challenges with regard to the way Danish people account for their perceived normality of drinking. It also shows that they have to prove their case and explain themselves if they choose to drink alcohol as recommended by the health authorities. This accentuates the importance of paying attention to focus group participant interaction when undertaking analysis of focus group data, recognising that individual viewpoints should not be isolated from the interaction that takes place between the participants.

Our analyses demonstrated how disagreements between participants can function as a catalyst to keep the focus group discussion going, while also moving perceptions from uncertainty to certainty about the health benefits of alcohol. In contrast, homogeneity in the groups (in this case age) resulted in general agreement and acceptance of the participants' utterances. Although some degree of homogeneity is favoured in order to create a positive atmosphere and promote group dynamics, the more homogenous, the greater the likelihood of polarisation (Sim, 1998). Therefore, the examples of how participants construct normality and disagree, and their important influence on the contents for this research, demonstrate that homogeneity must be challenged by ensuring a degree of heterogeneity to obtain data with sufficient variety. In order words, a group may be too homogeneous; thus influencing the range and variety of the data that emerges. This accentuates the role and responsibility of the moderator in being able to challenge the participants' accounts and ensure that all participants have the chance to express their views. It also highlights the delicate balance in composing a group that has enough in common to make discussion seem appropriate, yet sufficiently varying characteristics, experiences and perspectives to allow for divergent data to emerge through differences of opinion and debate (Barbour, 2007). Barbour (2007, pg. 81) further argues that rather than viewing disagreement as problem, the researcher needs to turn this into an advantage and use it as a resource in the analysis - in this case, obtaining a greater understanding of the different participants' perceptions about people's alcohol consumption and health.

Our analyses identified how interaction can come to a dead-end, including the risk of hierarchical issues. Based on the analyses from this study, the moderator's ability to pursue the participants' utterances may be the reason for coming to a dead-end. Hierarchical issues may also have emerged because of an interest in ensuring heterogeneity in the group construction. However, one aim of using focus groups is to bring together a diverse group to maximize exploration of different perspectives within the focus group setting (Kitzinger, 1994; Morgan, 1997). Morgan (1997) highlighted that the goal of focus group construction is homogeneity in background, e.g. age, and not homogeneity in attitudes. Thus, based on this discussion, and with the purpose of the study in mind, it was considered advantageous to consider both homogeneity and heterogeneity in the focus groups. In the case of dominant or expert participants in the groups, moderator control is encouraged. 
The interactional events stemming from our analyses led to considerations about the role and responsibility of the moderator. Although the role of the moderator and group interaction have been emphasized in the literature (Krueger et al., 2000; Morgan, 1997; Puchta et al., 2004; Bloor et al., 2001; Barbour, 2007), this paper links the interactional events to the influencing nature of the moderator's role. Our analyses demonstrated disagreement between participants in focus groups and how negotiations and lack of consensus impacted constructively on the content of the data that emerged. We emphasised the importance of paying attention to the interaction between participants for analytical purposes, but also for participants to feel comfortable in such cases where the interactions were characterised by dispreferred reactions. Thus, the moderator role involves thoroughly considering their responses to the participants' reactions and responses to others' conversations, and to signal approval and remain open to the interaction continuing, e.g. by keeping eye contact and nodding; listening and not commenting on the details of the discussion, or instead elaborating on aspects relevant to the study. This is particularly important when there are potential misunderstandings that could lead to deadends in discussion and the need to maintain the normative relation between adjacency pairs. In interactions characterised by a large degree of homogeneity; easy flow in the conversation and preferred reactions to other participants' utterances, the role of the moderator, may, besides paying close attention to the interaction, encompass challenging the participants' accounts to add new dimensions to the discussion or interrupt discussions in order to allow another viewpoint to emerge from a different participant or change the focus altogether.

In analysing focus group data from this research project, we chose conversation analysis as a source of inspiration. By choosing only elements of this analytical toolbox, we acknowledge that we have omitted substantial material. For example, conversation analysis, in its pure form, requires that transcripts of focus group data be produced according to a set of conventions, utilizing time-taking and a range of symbols to indicate specific features of talk (Puchta et al., 2004; Barbour, 2007). This, however, was not possible because the need for, and interest in, a more rigorous analysis of the group interaction emerged during thematic analysis of the data; after the focus groups had been transcribed. Thus, this paper does not take e.g. length of pauses into consideration. Bloor et al. (2001) claim, however, that some of these conventions can be omitted, and Barbour (2007) argues that even though a rigorous conversation analysis approach is not followed, the general focus group analysts can still learn from the attention given to the details of speech and interaction. We therefore argue that it has been appropriate to use only elements of conversation analysis in understanding group interaction in this research.

Because data are analysed in sequences, it can be argued that conversation analysis is a technical tool that does not take into consideration the "liveliness" of social interaction. Moreover, the fact that transcribed materials of focus group interactions is analysed (as opposed to for example video recordings of verbal and non-verbal language) may limit representation of forms of interaction (Halkier, 2010). However, analysing sequences of group interaction using adjacency pairs, preference organisation, accounts and so forth, allows and intensifies a different and comprehensive view of the data - allowing for perspectives that may not have emerged in the more traditional form of thematic analysis. For example, the interactional event of coming to a dead-end and making sense of it did not reveal itself as important for the content before analysing the exchanges of adjacency pairs. Thus, scrutinising data with a specific purpose, in this case group interaction, revealed a variety of interactional events that impacted on content, despite any limitations. 
We are aware that other analytical approaches could have been relevant in shedding light on group interaction in the empirical examples. For example, if we had used discursive psychology, the focus would have been on the discursive repertoires rather than solely the conversational situation itself (Potter, 1996; Puchta et al., 2004). One of the strategies in discourse psychology is factual descriptions in which participants construct their accounts as fact-like as possible. An example of factual descriptions from the empirical material is the interaction characterised by disagreement where participants for example use scientific knowledge about the liver to argue about alcohol and health. Thus, the interaction data of this research project could have been analysed using alternative tools; however, it is essential to ensure that the methodological tool is coherent with the knowledge interest of the research (Halkier, 2010).

The broad array of literature addressing focus group research indicates the usefulness of its application as a data collection method for qualitative research. Although the literature deals with focus group interaction as part of moderator questions, group composition and introduction to focus groups, less is available on the analysis of the social interaction in the group itself and the analytical outcome for the content of the data. Because focus groups are increasingly popular as a method of data collection, it is necessary that researchers critically reflect on and discuss the methodological advantages and limitations. This paper contributes to the body of knowledge on focus group interaction by exemplifying how interaction influences the data contents that emerge by using conversation analysis as inspiration. It builds on existing knowledge by illustrating four commonly occurring interactional events and considers the role of the moderator in case of such events. The paper emphasises that focusing on group interaction in the conduct and analysis of focus group interaction is essential to gain the full potential of the focus group method.

\section{References}

Barbour, R. S. (2007). Doing Focus Groups. London: SAGE.

Bloor, M., Frankland, J., Thomas, M., \& Robson, K. (2001). Focus Groups in Social Research. London: SAGE Publications.

Clark, J. M., Maben, J., \& Jones, K. (1996). The use of focus group interviews in nursing research: Issues and challenges. Nursing Times Research, 1, 143-153.

Creswell, J. W. (2003). Research design: Qualitative, quantitative, and mixed methods approaches. (2 ed.) Thousand Oaks: Sage Publications.

Curtis, E. \& Redmond, R. (2007). Focus groups in nursing research. Nurse Res., 14, 25-37.

Duggleby, W. (2005). What about focus group interaction data? Qualitative Health Research, 15, 832-840.

Gronbaek, M. (2001). Factors influencing the relation between alcohol and mortality - with focus on wine. Journal of Internal Medicine, 250, 291-308.

Grønkjær, M., Vinter-Larsen, M., Curtis, T., Grønbæk, M., \& Nørgaard, M. (2010). Alcohol use in Denmark: A descriptive study on drinking contexts. Addiction Research and Theory, 18, 359370 . 
Halkier, B. (2010). Focus groups as social enactments: integrating interaction and content in the analysis of focus group data. Qualitative Research, 10, 71-89.

Hydén, L. C. \& Bülow, P. H. (2003). Who's talking: drawing conclusions from focus groups: some methodological considerations. International Journal of Social Research Methodology, 6, 305321.

Kitzinger, J. (1995). Qualitative research. Introducing focus groups. BMJ, 311, 299-302.

Kitzinger, J. (1994). The methodology of Focus Groups: the importance of interaction between research participants. Sociology of Health and Illness, 16, 103-121.

Krueger, R. A. \& Casey, M. A. (2000). Focus groups: a practical guide for applied research. (3 ed.) Thousand Oaks: Sage Publication.

Mandag Morgen \& TrygFonden (2008). Fremtidens forebyggelse - ifølge danskerne [Prevention of the future-according to the Danes] Copenhagen: Mandag Morgen \& Trygfonden.

McLafferty, I. (2004). Focus group interviews as a data collecting strategy. Journal of Advanced Nursing, 48, 187-194.

Morgan, D. L. (1997). Focus groups as qualitative research. (2 ed.) Thousand Oaks: SAGE Publications.

Plummer-D'Amato, P. (2008). Focus group methodology part 1: considerations for design. International Journal of Therapy \& Rehabilitation, 15, 69-73.

Potter, J. (1996). Representing Reality. Discourse, Rhetoric and Social Construction. London: SAGE.

Puchta, C. \& Potter, J. (2004). Focus Group Practice. London: Sage Publications.

Rabiee, F. (2004). Focus-group interview and data analysis. Proc.Nutr.Soc., 63, 655-660.

Redmond, R. \& Curtis, E. (2009). Focus groups: principles and process. Nurse Researcher, 16, 5769.

Rose, G. (1992). The Strategy of Preventive Medicine. New York: Oxford University Press.

Silverman, D. (2006). Interpreting qualitative data. London: SAGE.

Sim, J. (1998). Collecting and analysing qualitative data: issues raised by the focus group. Journal of Advanced Nursing, 28, 345-352.

Warr, D. J. (2005). "It was fun... but we don't usually talk about these things": Analyzing Sociable Interaction in Focus Groups. Qualitative Inquiry, 11, 200-225.

WHO (2004). Global Status Report on Alcohol 2004 Geneva: World Health Organization, Department of Mental Health and Substance Abuse. 
Wibeck, V., Dahlgren, M. A., \& Oberg, G. (2007). Learning in focus groups: an analytical dimension for enhancing focus group research. Qualitative Research, 7, 249-267.

\section{Authors}

Mette Grønkjær works at the Clinical Nursing Research Unit, Aalborg Hospital, Aarhus University Hospital, Aalborg, Denmark. Tine Curtis works for Implement Consulting Group Hørsholm, Denmark. Charlotte de Crespigny works at the School of Population Health and Clinical Practice at the University of Adelaide, Australia. And Charlotte Delmar is employed at the Clinical Nursing Research Unit, Aalborg Hospital, Aarhus University Hospital, Aalborg, Denmark. Email for corresponding author (Grønkjær): mette.groenkjaer@rn.dk 